\title{
Identification of potential therapeutic target genes and mechanisms in head and neck squamous cell carcinoma by bioinformatics analysis
}

\author{
JING KUANG ${ }^{1}$, MEI ZHAO ${ }^{2}$, HUILIAN LI ${ }^{1}$, WEI DANG ${ }^{1}$ and WEI LI ${ }^{3}$ \\ Departments of ${ }^{1}$ Plastic Surgery, ${ }^{2}$ News Office and ${ }^{3}$ Healthcare, \\ Jinan Central Hospital Affiliated to Shandong University, Jinan, Shandong 250013, P.R. China
}

Received February 26, 2015; Accepted March 8, 2016

DOI: $10.3892 / \mathrm{ol} .2016 .4358$

\begin{abstract}
The present study aimed to identify the potential target genes and underlying molecular mechanisms involved in head and neck squamous cell carcinoma (HNSCC) by bioinformatics analysis. Microarray data of a Gene Expression Omnibus series GSE6631 was downloaded from the Gene Expression Omnibus database, which was generated from paired samples of HNSCC and normal tissue from 22 patients, and was used to identify differentially expressed genes (DEGs). Gene Ontology (GO) and Kyoto Encyclopedia of Genes and Genomes enrichment analyses were performed to investigate the functions of the identified DEGs. Furthermore, the protein-protein interaction (PPI) network of these DEGs was constructed using Cytoscape software. Between HNSCC and normal samples there was a difference in 419 DEGs, including 196 upregulated and 223 downregulated genes. The upregulated DEGs were mainly enriched in GO terms of cell adhesion, extracellular matrix (ECM) organization and collagen metabolic process, while the downregulated DEGs were mainly associated with epidermis development and epidermal cell differentiation. The DEGs were enriched in pathways such as ECM-receptor interaction, focal adhesion and drug metabolism. Fibronectin 1 (FN1), epidermal growth factor receptor (EGFR), collagen type I alpha 1 (COL1A1) and matrix metallopeptidase-9 (MMP-9) were hub nodes in the PPI network. These results suggested that cell adhesion and drug metabolism may be associated with HNSCC development, and genes such as FN1, EGFR, COL4A1 and MMP-9 may be potential therapeutic target genes in HNSCC.
\end{abstract}

Correspondence to: Dr Wei Li, Department of Healthcare, Jinan Central Hospital Affiliated to Shandong University, 105 Jiefang Road, Jinan, Shandong 250013, P.R. China

E-mail: weiliwlwl@163.com

Key words: head and neck squamous cell carcinoma, differentially expressed genes, molecular mechanism, bioinformatics analysis

\section{Introduction}

Head and neck squamous cell carcinoma (HNSCC) is the sixth most common type of cancer in the world (1). It is an epithelial cancer arising in the upper aerodigestive tract, including the pharynx, larynx and oral cavity (2). Furthermore, the head and neck region contains several distinct structures, such as the lips, nasopharynx, oropharynx and hypopharynx, which result in the large heterogeneity of $\operatorname{HNSCC}(2,3)$. In total, $>600,000$ novel cases of HNSCC are diagnosed annually (1). Currently, chemotherapy or radiotherapy with locoregional treatment is used for HNSCC patients $(4,5)$. However, the survival rate of this disease is only $40-50 \%$ within 5 years after diagnosis and treatment (6).

Numerous studies have explored the pathological mechanism underlying the development of $\operatorname{HNSCC}(7,8)$. Several genes have been identified to participate in the progression of HNSCC. For example,Zhang et al (9) reported that fos-related activator-1 could be used as a potential therapeutic target gene in oral squamous cell carcinoma, while transgelin 2 has an oncogenic function and may be regulated by the tumor suppressor microRNA-1 in HNSCC (7). Aberrant promoter methylation of the Nei endonuclease VIII-like 1 gene has a critical role in the progression and development of HNSCC (8). Certain signaling pathways have also been demonstrated to be important in HNSCC. For example, Pedrero et al (10) reported that dysregulation of the phosphatidylinositol-4,5-bisphosphate 3-kinase/AKT/phosphatase and tensin homolog signaling pathway may contribute to early HNSCC tumorigenesis. In addition, cyclooxygenase-2 (COX-2) signaling pathway is closely associated with tumor angiogenesis in HNSCC, and COX-2 overexpression predicts a shorter survival in patients with head and neck cancer (11). The coactivation of the mitogen-activated protein kinase and I $\mathrm{B}$ kinase signaling pathways may suppress the mechanism of signal transduction by regulating the secretion of interleukin- 8 and vascular endothelial growth factor in human HNSCC (12). Although numerous factors have been identified to contribute to HNSCC, the pathogenic mechanisms of HNSCC remain to be clearly demonstrated in order to identify potential target genes for the treatment of HNSCC.

In the present study, the differentially expressed genes (DEGs) between HNSCC and normal samples were analyzed 
to gain a better insight of HNSCC. Gene Ontology (GO) and Kyoto Encyclopedia of Genes and Genomes (KEGG) enrichment analyses of DEGs were performed, and the protein-protein interaction (PPI) network of these DEGs was constructed. The purpose of the present study was to explore the underlying mechanisms of HNSCC and to identify novel potential target genes for HNSCC therapy.

\section{Materials and methods}

Affymetrix microarray data. Gene Expression Omnibus (GEO; www.ncbi.nlm.nih.gov/geo/) is a database repository of high throughput gene expression data, which segregates data into three principle components: Platform (GPL), series (GSE) and sample (GSM). The array data of GSE6631, based on the GPL8300 Affymetrix Human Genome U95 Version 2 Array platform (Affymetrix, Inc., Santa Clara, CA, USA) was downloaded from the GEO database, which was deposited by Kuriakose et al (13). The dataset was generated from paired (from the same patient) samples of tumor and normal tissues from 22 patients with histologically confirmed HNSCC by Kuriakose et al (13).

Data preprocessing and DEGs analysis. The original probe-level data in CEL files (raw probe level data) were converted into gene expression values. Data were normalized using the Bioconductor R package affy version 1.32.0 (Affymetrix, Inc., Santa Clara, CA, USA) (14). Nonspecific probes were filtered. If multiple probes corresponded to the same gene, the average expression value was calculated to represent the expression levels of that gene. The samr package (version 2.0; cran.r-project.org/web/packages/samr/index. html) in R (www.r-project.org/) (15) was applied to identify DEGs between HNSCC and normal samples. $\Delta=1.3$ and fold-change $>1.5$ were used as the cutoff criteria, based on the experience of the present authors.

Functional enrichment analysis of DEGs. The GO database (geneontology.org/page/go-database) (16) is a collection of numerous gene annotation terms. The knowledge contained in the KEGG database (www.genome.jp/kegg/) (17) was applied to identify functional and metabolic pathways. The Database for Annotation, Visualization and Integrated Discovery (DAVID) version 6.7 (National Cancer Institute at Frederick, Frederick, MD, USA) (18) was used as a gene functional enrichment analysis tool to understand the biological meaning of the results of bioinformatics analysis. GO and KEGG enrichment analyses for the upregulated and downregulated identified DEGs were performed with DAVID. P $<0.05$ and false discovery rate $<0.01$ were selected as the cutoff criteria.

Construction of PPI network and disease enrichment analysis. The Search Tool for the Retrieval of Interacting Genes/Proteins (version 9.05; string-db.org) (19) is an online database that contains comprehensive information of proteins. This online tool was applied to analyze the interactions of protein pairs. PPI network of DEGs was constructed using Cytoscape software (version 3.0.1; Cytoscape Consortium San Diego, CA, USA) (20). The degree of connectivity was analyzed and used to obtain the hub proteins in the PPI network.
A

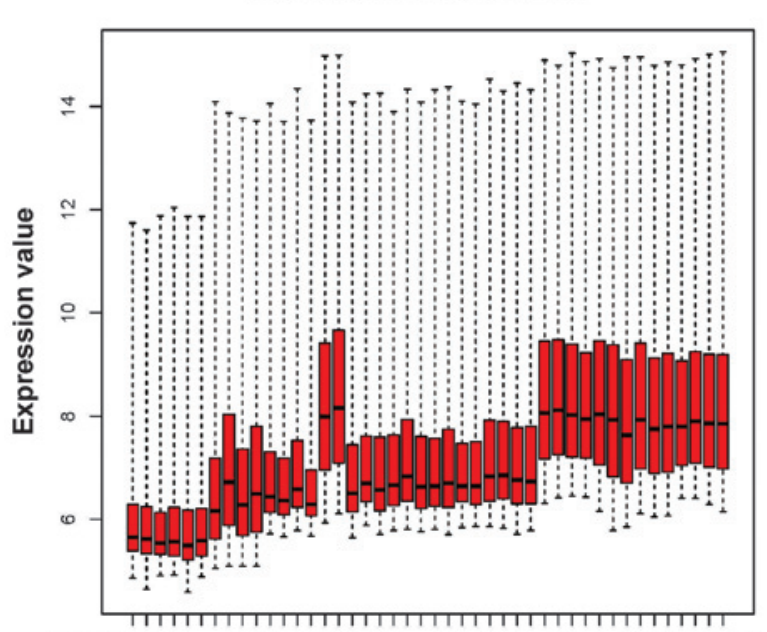

GSM153813.CEL.gz GSM153827.CEL.gz GSM153841.CEL.gz GSM153855.CEL.gz

Sample list

B

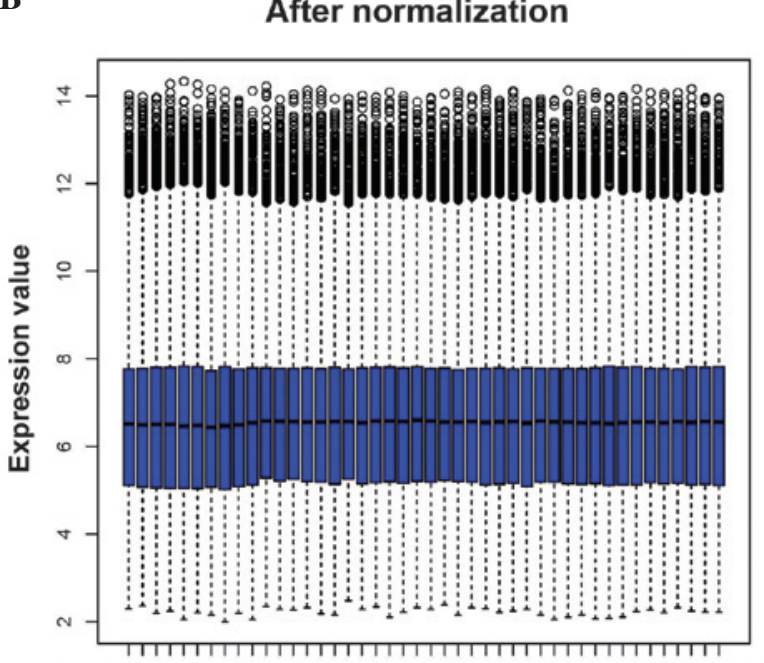

GSM153813.CEL.gz GSM153827.CEL.gz GSM153841.CEL.gz GSM153855.CEL.gz

Sample list

Figure 1. Box plots of data (A) before (red) and (B) after (blue) normalization. The $\mathrm{x}$ axis represents the samples from the microarray data, while the $\mathrm{y}$ axis represents the gene expression values. The box plot refers to the interquartile range (25-75\%), and the median is shown as a black horizontal line.

\section{Results}

Identification of DEGs. As represented in Fig. 1, the raw expression data were preprocessed and normalized. A total of 419 DEGs were identified between HNSCC and normal samples, including 196 upregulated genes and 223 downregulated genes.

Functional enrichment analysis of DEGs. A total of $39 \mathrm{GO}$ terms of upregulated and downregulated DEGs were obtained. The top 5 GO terms of upregulated and downregulated genes are indicated in Table I. The upregulated DEGs were significantly associated with cell adhesion, extracellular matrix (ECM) organization, collagen metabolic process and proteinaceous ECM, while the downregulated genes were mainly involved in epidermis development, ectoderm development and epidermal cell differentiation. 
Table I. GO terms most frequently enriched by upregulated and downregulated DEGs in head and neck squamous cell carcinoma.

\begin{tabular}{|c|c|c|c|c|}
\hline Category & Term & Count $^{\mathrm{a}}$ & P-value & FDR \\
\hline \multicolumn{5}{|l|}{ Upregulated DEGs } \\
\hline GOTERM_BP & GO:0007155 cell adhesion & 42 & $4.92 \mathrm{E}-17$ & $8.08 \mathrm{E}-14$ \\
\hline GOTERM_BP & GO:0022610 biological adhesion & 42 & $5.18 \mathrm{E}-17$ & $8.50 \mathrm{E}-14$ \\
\hline GOTERM_BP & GO:0030198 extracellular matrix organization & 17 & $2.12 \mathrm{E}-13$ & $3.48 \mathrm{E}-10$ \\
\hline GOTERM_BP & GO:0043062 extracellular structure organization & 19 & $2.20 \mathrm{E}-12$ & $3.62 \mathrm{E}-09$ \\
\hline GOTERM_BP & GO:0032963 collagen metabolic process & 10 & $3.75 \mathrm{E}-11$ & $6.15 \mathrm{E}-08$ \\
\hline GOTERM_CC & GO:0005578 proteinaceous extracellular matrix & 40 & $1.88 \mathrm{E}-26$ & $2.48 \mathrm{E}-23$ \\
\hline GOTERM_CC & GO:0031012 extracellular matrix & 40 & $3.23 \mathrm{E}-25$ & 4.27E-22 \\
\hline GOTERM_CC & GO:0044420 extracellular matrix part & 25 & $5.83 \mathrm{E}-22$ & $7.70 \mathrm{E}-19$ \\
\hline GOTERM_CC & GO:0044421 extracellular region part & 56 & $4.52 \mathrm{E}-21$ & $5.97 \mathrm{E}-18$ \\
\hline GOTERM_CC & GO:0005581 collagen & 14 & $3.91 \mathrm{E}-16$ & $5.88 \mathrm{E}-13$ \\
\hline GOTERM_MF & GO:0005201 extracellular matrix structural constituent & 15 & $3.11 \mathrm{E}-12$ & 4.32E-09 \\
\hline GOTERM_MF & GO:0050840 extracellular matrix binding & 8 & $3.24 \mathrm{E}-08$ & 4.50E-05 \\
\hline GOTERM_MF & GO:0005198 structural molecule activity & 27 & $9.82 \mathrm{E}-08$ & $1.36 \mathrm{E}-04$ \\
\hline GOTERM_MF & GO:0005509 calcium ion binding & 32 & $4.02 \mathrm{E}-07$ & $5.58 \mathrm{E}-04$ \\
\hline GOTERM_MF & GO:0005518 collagen binding & 7 & $5.28 \mathrm{E}-06$ & 7.33E-03 \\
\hline \multicolumn{5}{|c|}{ Downregulated DEGs } \\
\hline GOTERM_BP & GO:0008544 epidermis development & 20 & $1.27 \mathrm{E}-11$ & 2.11E-08 \\
\hline GOTERM_BP & GO:0007398 ectoderm development & 20 & $5.04 \mathrm{E}-11$ & 8.39E-08 \\
\hline GOTERM_BP & GO:0009913 epidermal cell differentiation & 13 & $3.26 \mathrm{E}-10$ & $5.42 \mathrm{E}-07$ \\
\hline GOTERM_BP & GO:0030855 epithelial cell differentiation & 15 & 8.05E-09 & $1.34 \mathrm{E}-05$ \\
\hline GOTERM_BP & GO:0030216 keratinocyte differentiation & 11 & $2.64 \mathrm{E}-08$ & 4.40E-05 \\
\hline GOTERM_CC & GO:0001533 cornified envelope & 9 & $3.27 \mathrm{E}-10$ & 4.24E-07 \\
\hline GOTERM_MF & GO:0005198 structural molecule activity & 26 & $5.36 \mathrm{E}-06$ & $7.55 \mathrm{E}-03$ \\
\hline
\end{tabular}

${ }^{a}$ Enriched gene number in the GO category. GO, Gene Ontology; DEGs, differentially expressed genes; BP, biological process; CC, cellular component; MF, molecular function; FDR, false discovery rate.

Table II. KEGG pathway enrichment analysis of upregulated and downregulated differentially expressed genes.

\begin{tabular}{|c|c|c|c|c|}
\hline KEGG pathway term & Count $^{\mathrm{a}}$ & Genes & P-value & FDR \\
\hline \multicolumn{5}{|l|}{ Upregulated genes } \\
\hline ECM-receptor interaction & 23 & COL1A1, COL4A1, TNC & $1.24 \mathrm{E}-20$ & $1.35 \mathrm{E}-17$ \\
\hline Focal adhesion & 26 & FN1, EGFR, COL1A1 & $4.22 \mathrm{E}-15$ & $4.59 \mathrm{E}-12$ \\
\hline Small cell lung cancer & 11 & FN1, CKS1B, LAMB3, COL4A2 & $2.11 \mathrm{E}-06$ & 0.00229 \\
\hline \multicolumn{5}{|l|}{ Downregulated genes } \\
\hline Drug metabolism & 9 & CYP3A5, CYP2C18, FMO2, MAOB & $6.49 \mathrm{E}-06$ & 0.0071 \\
\hline
\end{tabular}

${ }^{a}$ Enriched gene number in the KEGG pathway term. KEGG, Kyoto Encyclopedia of Genes and Genomes; FDR, false discovery rate; ECM, extracellular matrix; COLA, collagen type alpha; TNC, tenascin C; FN1, fibronectin 1; EGFR, epidermal growth factor receptor; CKS1B, cyclin-dependent kinases regulatory subunit 1; LAMB3, laminin, beta 3; CYP, cytochrome P450; FMO, flavin containing monooxygenase 2; MAOB, monoamine oxidase B.

The pathways of these upregulated and downregulated genes are indicated in Table II. The upregulated genes were mainly involved in ECM-receptor interaction, focal adhesion and small cell lung cancer. Genes such as fibronectin 1 (FN1), epidermal growth factor receptor (EGFR) and collagen type I alpha 1 (COL1A1) were identified in the focal adhesion pathway. By contrast, the downregulated DEGs were enriched in drug metabolism. Cytochrome P450 3A5 (CYP3A5) was identified in the drug metabolism pathway.

PPI network construction and disease enrichment analysis. The results of the PPI network analysis are represented in 


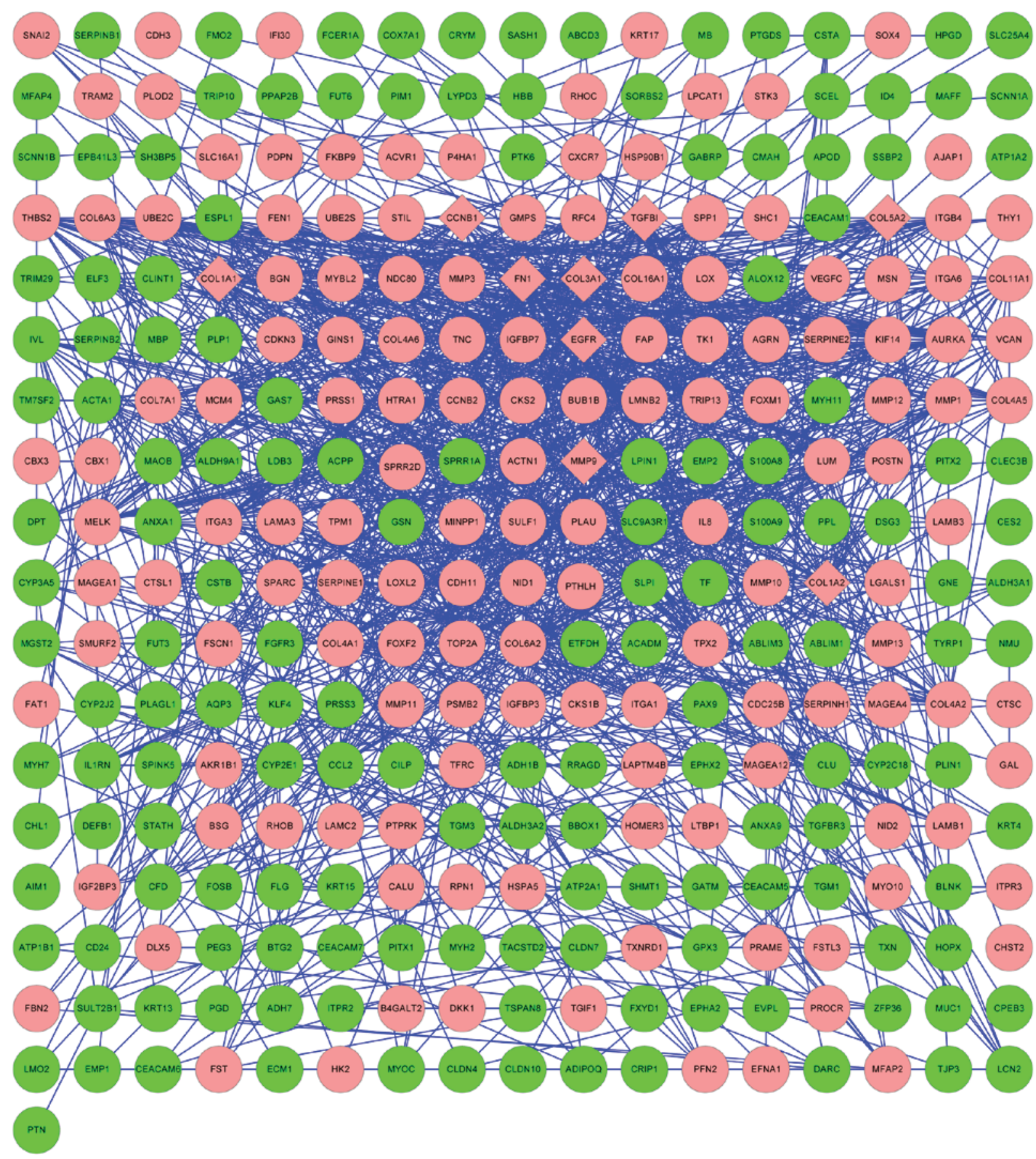

Figure 2. Protein-protein interaction network for differentially expressed genes. Red nodes correspond to upregulated genes in HNSCC, while green nodes correspond to downregulated genes in HNSCC. Diamonds represent hub nodes (nodes with a degree of connectivity $\geq 30$ ). HNSCC, head and neck squamous cell carcinoma.

Fig. 2. The upregulated genes FN1, EGFR, COL1A1, matrix metallopeptidase-9 (MMP-9), COL5A2, COL1A2, COL3A1, transforming growth factor, beta-induced and cyclin $\mathrm{B} 1$ were selected as hub nodes.

\section{Discussion}

In the present study, gene expression profile data were downloaded from the GEO database to identify DEGs in HNSCC using bioinformatics analysis. A total of 419 DEGs between HNSCC and normal samples, including 196 upregulated and 223 downregulated genes, were selected. The results of functional enrichment analysis revealed that the upregulated genes, including FN1, EGFR and COL1A1, were associated with GO term of cell adhesion, while the downregulated DEGs, including CYP3A5, were enriched in drug metabolism pathways. According to the results of the PPI network analysis, FN1, EGFR, COL1A1 and MMP-9 were identified as hub nodes. Therefore, these DEGs and their interacting patners may be involved in HNSCC development.

Cell adhesion is the process of binding of a cell to a surface or substrate, such as the ECM or another cell (21). In 
the present study, the majority of the upregulated DEGs were enriched in pathways of ECM-receptor interaction and focal adhesion. Previous studies have indicated that ECM-receptor interaction and focal adhesion were associated with cell adhesion (22). Recent evidence suggests that cell adhesion is mediated by several genes, including FN1, EGFR and COL4A1 (23-25). FN1 is an ECM glycoprotein (26) involved in cell adhesion (27), which corresponds to the pathway identified in the present study. It was previously reported that FN1 acts as a tumor suppressor gene, playing a critical role in migration and invasion of laryngeal carcinoma (23), which is the most common type of HNSCC (28). EGFR was also indicated to be associated with HNSCC (29). EGFR is the cell-surface receptor of the EGF family (30). In the present study, EGFR was enriched in GO terms of cell adhesion and pathway of focal adhesion, which was consistent with previous studies that reported that EGFR contributed to transduce extracellular signals to intracellular responses, thus influencing adhesion and proliferation in tumor cells $(24,31)$. Rubin Grandis et al (32) reported that EGFR was overexpressed in HNSCC. High expression levels of EGFR have been associated with reduced survival and increased risk of recurrence in HNSCC (33). COL4A1 is a member of the collagen family, and is also associated with cell adhesion (25). The adhesion of cells to collagen is mediated by fibronectin (25). Tanaka et al (34) indicated that the differential expression of type IV collagen chains was associated with the invasive potential of cell carcinoma. The results of the present study indicated that FN1, EGFR and COL4A1 were upregulated genes in HNSCC and hub nodes in the PPI network, which suggests that FN1, EGFR and COL4A1 may regulate cell adhesion in HNSCC. Thus, cell adhesion may participate in HNSCC through multiple genes, including FN1, EGFR and COL4A1, which may be potential therapeutic target genes in HNSCC.

In the present study, the downregulated DEGs such as CYP3A5, were significantly enriched in the pathway of drug metabolism ( $\mathrm{P}=6.49 \mathrm{E}-06)$. CYP3A5 encodes a member of the cytochrome P450 superfamily of enzymes (34). It has been reported that cytochrome $\mathrm{P} 450$ proteins catalyze multiple reactions, including drug metabolism (35). Olivieri et al (36) reported that cytochrome $\mathrm{P} 450$ gene polymorphisms were important in the tumorigenesis and progression of HNSCC (36). These results suggested that CYP3A5 may regulate HNSCC development through the drug metabolism pathway. Therefore, this pathway may be associated with HNSCC progression.

In addition to FN1, EGFR and COL4A1, MMP-9 was also identified as a hub node in the present PPI network analysis. MMP-9 is an enzyme that belongs to the MMP family (35). It has been reported that MMPs participate in cancer invasion and metastasis (37). In the present study, MMP-9 was an upregulated gene, which was consistent with previous studies $(38,39)$. For example, Riedel et al (38) reported that the expression levels of MMP-9 were significantly higher in HNSCC patients than in healthy individuals. MMP-9 regulates cell proliferation through modulating the nuclear factor- $\kappa \mathrm{B}$ signaling pathway in HNSCC (40). Furthermore, MMP-9 was associated with cancer in the present study. Thus, MMP-9 may be a potential target gene for the treatment of HNSCC.
In conclusion, a total of 419 DEGs were identified between HNSCC and normal samples, and the present study indicates that cell adhesion and drug metabolism may be closely associated with HNSCC development. Genes such as FN1, EGFR, COL4A1 and MMP-9 may be potential therapeutic target genes in HNSCC. However, further studies are required to confirm the present results.

\section{References}

1. Ferlay J, Shin HR, Bray F, Forman D, Mathers C and Parkin DM: Estimates of worldwide burden of cancer in 2008: GLOBOCAN 2008. Int J Cancer 127: 2893-2917, 2010.

2. Rothenberg SM and Ellisen LW: The molecular pathogenesis of head and neck squamous cell carcinoma. J Clin Invest 122: 1951-1957, 2012.

3. Hasina R, Whipple ME, Martin LE, Kuo WP, Ohno-Machado L and Lingen MW: Angiogenic heterogeneity in head and neck squamous cell carcinoma: Biological and therapeutic implications. Lab Invest 88: 342-353, 2008.

4. Belcher R, Hayes K, Fedewa S and Chen AY: Current treatment of head and neck squamous cell cancer. J Surg Oncol 110: 551-574, 2014.

5. Colevas AD: Chemotherapy options for patients with metastatic or recurrent squamous cell carcinoma of the head and neck. J Clin Oncol 24: 2644-2652, 2006.

6. Leemans CR, Braakhuis BJ and Brakenhoff RH: The molecular biology of head and neck cancer. Nat Rev Cancer 11: 9-22, 2011.

7. Nohata N, Sone Y, Hanazawa T, Fuse M, Kikkawa N, Yoshino H, Chiyomaru T, Kawakami K, Enokida H, Nakagawa M, et al: miR-1 as a tumor suppressive microRNA targeting TAGLN2 in head and neck squamous cell carcinoma. Oncotarget 2: 29-42, 2011.

8. Chaisaingmongkol J, Popanda O, Warta R, Dyckhoff G, Herpel E, Geiselhart L, Claus R, Lasitschka F, Campos B, Oakes CC, et al: Epigenetic screen of human DNA repair genes identifies aberrant promoter methylation of NEIL1 in head and neck squamous cell carcinoma. Oncogene 31: 5108-5116, 2012.

9. Zhang L, Pan HY, Zhong LP, Wei KJ, Yang X, Li J, Shen GF and Zhang Z: Fos-related activator-1 is overexpressed in oralsquamous cell carcinoma and associated with tumor lymph node metastasis. J Oral Pathol Med 39: 470-476, 2010.

10. Pedrero JM, Carracedo DG, Pinto CM, Zapatero AH, Rodrigo JP Nieto CS and Gonzalez MV: Frequent genetic and biochemical alterations of the PI3-K/AKT/PTEN pathway in head and neck squamous cell carcinoma. Int J Cancer 114: 242-248, 2005.

11. Gallo O, Masini E, Bianchi B, Bruschini L, Paglierani M and Franchi A: Prognostic significance of cyclooxygenase-2 pathway and angiogenesis in head and neck squamous cell carcinoma. Hum Pathol 33: 708-714, 2002.

12. Bancroft CC, Chen Z, Dong G, Sunwoo JB, Yeh N, Park C and Van Waes C: Coexpression of proangiogenic factors IL-8 and VEGF by human head and neck squamous cell carcinoma involves coactivation by MEK-MAPK and IKK-NF-kappaB signal pathways. Clin Cancer Res 7: 435-442, 2001.

13. Kuriakose MA, Chen WT,HeZM, Sikora AG,Zhang P,Zhang ZY, Qiu WL, Hsu DF, McMunn-Coffran C, Brown SM, et al: Selection and validation of differentially expressed genes in head and neck cancer. Cell Mol Life Sci 61: 1372-1383, 2004.

14. Gautier L, Cope L, Bolstad BM and Irizarry RA: affy - analysis of Affymetrix GeneChip data at the probe level. Bioinformatics 20: 307-315, 2004.

15. Tusher VG, Tibshirani R and Chu G: Significance analysis of microarrays applied to the ionizing radiation response. Proc Natl Acad Sci USA 98: 5116-5121, 2001.

16. Ashburner M, Ball CA, Blake JA, Botstein D, Butler H, Cherry JM, Davis AP, Dolinski K, Dwight SS, Eppig JT, et al; The Gene Ontology Consortium: Gene Ontology: Tool for the unification of biology. Nat Genet 25: 25-29, 2000.

17. Altermann E and Klaenhammer TR: PathwayVoyager: Pathway mapping using the Kyoto Encyclopedia of Genes and Genomes (KEGG) database. BMC Genomics 6: 60, 2005.

18. Huang DW, Sherman BT, Tan Q, Kir J, Liu D, Bryant D, Guo Y, Stephens R, Baseler MW, Lane HC and Lempicki RA: DAVID Bioinformatics Resources: Expanded annotation database and novel algorithms to better extract biology from large gene lists. Nucleic Acids Res 35 (Web Server issue): W169-W175, 2007. 
19. Szklarczyk D, Franceschini A, Kuhn M, Simonovic M, Roth A, Minguez P, Doerks T, Stark M, Muller J, Bork P, et al: The STRING database in 2011: Functional interaction networks of proteins, globally integrated and scored. Nucleic Acids Res 39 (Database issue): D561-D568, 2011.

20. Smoot ME, Ono K, Ruscheinski J, Wang PL and Ideker T: Cytoscape 2.8: New features for data integration and network visualization. Bioinformatics 27: 431-432, 2011.

21. Gumbiner BM: Cell adhesion: The molecular basis of tissue architecture and morphogenesis. Cell 84: 345-357, 1996.

22. Albelda SM and Buck CA: Integrins and other cell adhesion molecules. FASEB J 4: 2868-2880, 1990.

23. Wang F, Song G, Liu M, Li X and Tang H: miRNA-1 targets fibronectin 1 and suppresses the migration and invasion of the HEp2 laryngeal squamous carcinoma cell line. FEBS Lett 585 3263-3269, 2011.

24. Goerner M, Seiwert TY and Sudhoff H: Molecular targeted therapies in head and neck cancer - an update of recent developments. Head Neck Oncol 2: 8, 2010.

25. Kleinman HK, Martin GR and Fishman PH: Ganglioside inhibition of fibronectin-mediated cell adhesion to collagen. Proc Natl Acad Sci USA 76: 3367-3371, 1979.

26. Pankov R and Yamada KM: Fibronectin at a glance. J Cell Sci 115: 3861-3863, 2002

27. Soikkeli J, Podlasz P, Yin M, Nummela P, Jahkola T, Virolainen S, Krogerus L, Heikkilä P, von Smitten K, Saksela O and Hölttä E: Metastatic outgrowth encompasses COL-I, FN1, and POSTN up-regulation and assembly to fibrillar networks regulating cell adhesion, migration, and growth. Am J Pathol 177: 387-403, 2010.

28. Mao L, Hong WK and Papadimitrakopoulou VA: Focus on head and neck cancer. Cancer Cell 5: 311-316, 2004.

29. Erjala K, Sundvall M, Junttila TT, Zhang N, Savisalo M, Mali P, Kulmala J, Pulkkinen J, Grenman R and Elenius K: Signaling via ErbB2 and ErbB3 associates with resistance and epidermal growth factor receptor (EGFR) amplification with sensitivity to EGFR inhibitor gefitinib in head and neck squamous cell carcinoma cells. Clin Cancer Res 12: 4103-4111, 2006.

30. Herbst RS: Review of epidermal growth factor receptor biology. Int J Radiat Oncol Biol Phys 59 (Suppl 2): S21-S26, 2004.
31. Rocha-Lima CM, Soares HP, Raez LE and Singal R: EGFR targeting of solid tumors. Cancer Control 14: 295-304, 2007.

32. Rubin Grandis J, Melhem MF, Gooding WE, Day R, Holst VA, Wagener MM, Drenning SD and Tweardy DJ: Levels of TGF-alpha and EGFR protein in head and neck squamous cell carcinoma and patient survival. J Natl Cancer Inst 90: 824-832, 1998.

33. Bei R, Budillon A, Masuelli L, Cereda V, Vitolo D, Di Gennaro E, Ripavecchia V, Palumbo C, Ionna F, Losito S, et al: Frequent overexpression of multiple ErbB receptors by head and neck squamous cell carcinoma contrasts with rare antibody immunity in patients. J Pathol 204: 317-325, 2004.

34. Tanaka K, Iyama K, Kitaoka M, Ninomiya Y, Oohashi T, Sado Y and Ono T: Differential expression of alpha 1(IV), alpha 2(IV), alpha 5(IV) and-alpha 6(IV) collagen chains in the basement membrane of basal cell carcinoma. Histochem J 29: 563-570, 1997.

35. Bains RK, Kovacevic M, Plaster CA, Tarekegn A, Bekele E, Bradman NN and Thomas MG: Molecular diversity and population structure at the cytochrome P450 3A5 gene in Africa. BMC Genet 14: 34, 2013.

36. Olivieri EH, da Silva SD, Mendonça FF, Urata YN, Vidal DO, Faria MA, Nishimoto IN, Rainho CA, Kowalski LP and Rogatto SR: CYP1A2*1C, CYP2E1*5B, and GSTM1 polymorphisms are predictors of risk and poor outcome in head and neck squamous cell carcinoma patients. Oral Oncol 45: e73-e79, 2009.

37. Curran S and Murray GI: Matrix metalloproteinases: Molecular aspects of their roles in tumour invasion and metastasis. Eur J Cancer 36: 1621-1630, 2000.

38. Riedel F, Götte K, Schwalb J and Hörmann K: Serum levels of matrix metalloproteinase-2 and -9 in patients with head and neck squamous cell carcinoma. Anticancer Res 20: 3045-3049, 2000.

39. Sinpitaksakul SN, Pimkhaokham A, Sanchavanakit N and Pavasant P: TGF- $\beta 1$ induced MMP-9 expression in HNSCC cell lines via Smad/MLCK pathway. Biochem Biophys Res Commun 371: 713-718, 2008.

40. Aggarwal S, Takada Y, Singh S, Myers JN and Aggarwal BB: Inhibition of growth and survival of human head and neck squamous cell carcinoma cells by curcumin via modulation of nuclear factor-kappaB signaling. Int J Cancer 111: 679-692, 2004. 